International Journal of Engineering \& Technology, $7(2.26)(2018) 4-6$
International Journal of Engineering \& Technology
SPC
Website: www.sciencepubco.com/index.php/IJET
Research paper

\title{
Green cognitive communication approaches
}

\author{
M. Devi ${ }^{1}$ *, M. Thalaimalaichamy ${ }^{1}$, G. Usha ${ }^{1}$, K. T. Panneerselvam ${ }^{1}$, M. Suba ${ }^{1}$, M. Venkateshkumar ${ }^{1}$ \\ ${ }^{1}$ Department of ECE, School of EEE, SRC, SASTRA University, Kumbakonam \\ *Corresponding author E-mail: thalaimalaichamy@src.sastra.edu
}

\begin{abstract}
Generally one of the reasons for global warming is Co2 emission. The rapid growth of wireless communication is the reason for more emission. This creates more health issues, especially children. To reduce the issues, now the wireless communication is tilted towards green communication. Green communication cannot be achieved in single step. In this paper some of techniques are followed to achieve sustainable communication. The main objective of this paper is cognitive radio (CR), which makes use of spectrum in efficient way. In our proposed system, we focused on green cognitive cycle, Cognitive radio-Ultra wide band (CR-UWB) and Cognitive radio-Orthogonal Frequency Division Multiplexing (CR-OFDM). With these above methods we achieved better achievements in terms of efficiency, less time delay, resource utilization and low power for $5 \mathrm{G}$ wireless green communication.
\end{abstract}

Keywords: Cognitive Radio; Sharing; UWB; OFDM; Power Spectral Density (PSD

\section{Introduction}

The green communication is achieved by energy reductions, power consumption, very lower electromagnetic (EM) radiation, proper utilization of resources/resource consumption and minimizing the environmental impacts. Cognitive radio (1) is an intelligent radio whose functions are sensing, management, sharing and hand-off. CR senses the spectrum through adaptive learning (4) (intelligent) and finds the best unoccupied spectrum from available spectrum. After sensing, secondary (unlicensed) users are managed (1) to idle spectrum where the primary users are unavailable. Then the secondary user shares the spectrum with other primary users. Whenever the primary wants their spectrum, the secondary should vacate and hand-offed (8) to another idle channel.CR itself makes the resources to utilize in efficient way that changes the path towards green communication. This paper is organized as follows, section 2 describes green cognitive cycle, section 3 discusses the CR-OFDM and the summary of CR-UWB is provided in section 4 . Finally the paper is concluded in section 5.

\section{Green cognitive cycle}

In our proposed system CR's cyclelis slight lyre-formed as green cognitive cycle and the functions are modified as group sensing, bunch management, channel partitioning (triple and twin sharing) and band hand-off. Green cognitive functions enrich the green communication better than CR. Figure 1shown below is green cognitive cycle.

\subsection{Adaptive group sensing}

Before sensing the spectrum, best sensing method should be adapted. In the real time, system one best method may not be pointed out for sensing, so the optimum is analyzed through fuzzy. In group sensing (3), CR's spectrum sensing (4),(5),(6),(7) is changed to group spectrum scanning which identify group of idle channel and the length of the group is adaptive. Due to this CR's power is not wasted. Energy can be utilized in a proper way.

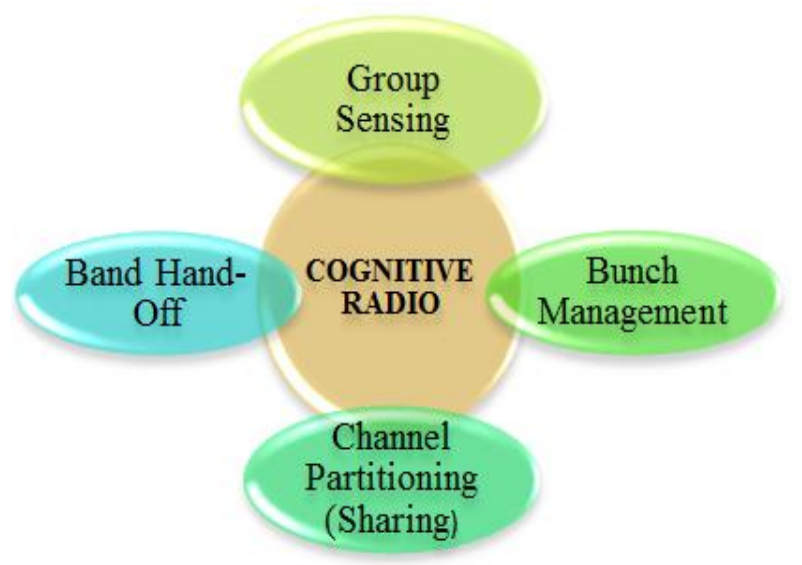

Fig. 1: Green Cognitive Cycle.

\subsection{Bunch management}

A group of vacant spectrum is sensed by $\mathrm{CR}$, from that a bunch of secondary's can occupy that idle group of vacant spectrum. Best group among the idle groups is identified by fuzzy and then the best group can be leased for high cost payee.

Table 1: Sense \& Manage (S \& M) Elapse Time for Various SNR

\begin{tabular}{llll}
\hline SNR S \& M-Elapse time & $-5 \mathrm{~dB}$ & $0 \mathrm{~dB}$ & $20 \mathrm{~dB}$ \\
\hline Single Slot & $0.341524 \mathrm{~s}$ & $0.275913 \mathrm{~s}$ & $0.298558 \mathrm{~s}$ \\
Double Slots & $0.172181 \mathrm{~s}$ & $0.146425 \mathrm{~s}$ & $0.151587 \mathrm{~s}$ \\
Eight Slots & $0.071700 \mathrm{~s}$ & $0.055009 \mathrm{~s}$ & $0.056455 \mathrm{~s}$ \\
\hline
\end{tabular}

In the above Table 1, sensing and management of single, double and eight channels were performed; the result shows that $S \& M$ of Eight slots elapse time is less than single and double slots. With 
5dBSNR, eight slots elapsed time is $0.071700 \mathrm{~s}$ and for single slot time is $0.341524 \mathrm{~s}$ which is higher than the elapse time of eight slots. Similarly for SNR of $20 \mathrm{~dB}$, elapse time of single slotis $0.298558 \mathrm{~s}$ and it is for eight slots are $0.056455 \mathrm{~s}$, comparatively eight slots elapsed time is low.

\subsection{Channel partitioning}

In channel partitioning, a single channel is partitioned in to subspectrum and then each sub-slot is given to separate secondary user. Due to this narrow band of secondary users (SUs) are possible to occupy the same channel with guard bands to avoid interference. This may leads to utilization of channel resource in an efficient way. When two/three users share the same channel then it is described as twin/triple sharing consecutively.

\subsection{Band hand-off}

When the primary(s) needs their slot, then the occupied secondary group should be handed over to new idle group of spectrum or partitioned channel slots has to be handed over to new partitioned channel slots. The Authors proposed a novel spectrum hand-off approach and algorithm (8),(9) and Spectrum mobility (10) of cognitive radio networks.

\section{CR-OFDM}

Nowadays, OFDM techniques are implemented in most of the existing wireless communication systems. OFDM's capacities such as sensing and shaping with its flexibility and adaptability make the OFDM technique suitable for CR system (13). Two methods of spectrum selections are described in CROFDM.OFDM is a multicarrier modulation technique which can overcome many problems like high bit rate, time dispersion and Inter-symbol interference (ISI).It minimizes the effect of multipath channels dispersion, reduces the need for complex equalizers and also extends the cyclic prefix (CP) to reduce ISI. Moreover the benefits of OFDM comprises of high spectral efficiency, flexible bandwidths enabled through scalability, robustness against narrowband interference (NBI) and effective implementation using Fast Fourier transform (FFT).

Method 1 of CR-OFDM is shown in Figure 2, in which the CR first scans the spectrum and identifies active PUs and spectrum holes. In OFDM system after performing serial to parallel ( $\mathrm{S}$ to $\mathrm{P}$ ) conversion, sensed information of $\mathrm{CR}$ is given as the input for

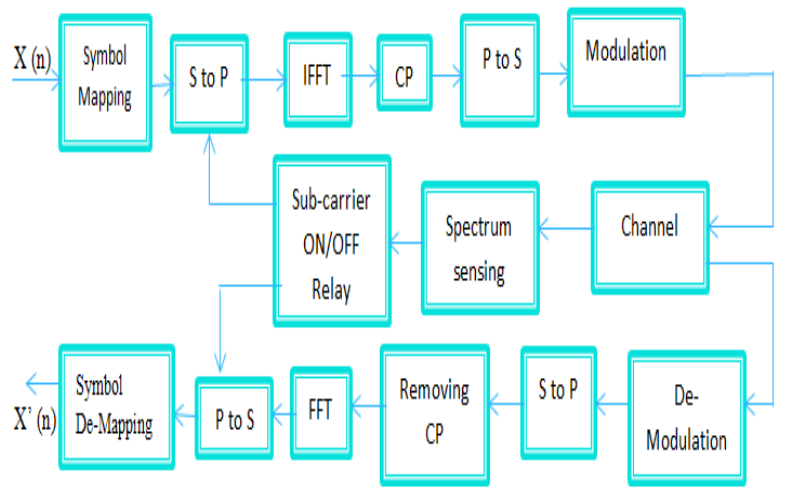

Fig. 2: CR-OFDM.

OFDM System to Shapes and Masks (Turned Off) Some Subcarriers which Cause Interference to Pus. Figure 3 Shows the Schematic

Diagram of secondary OFDM signal and PUs, specific subcarriers of OFDM signals are turned off wherever PUs are present. Later the OFDM signals are allocated to unoccupied band of spectrum.
Subcarrier selection is shown in Figure 4. The same inverse process should be repeated in the receiver side to recover the original signal.

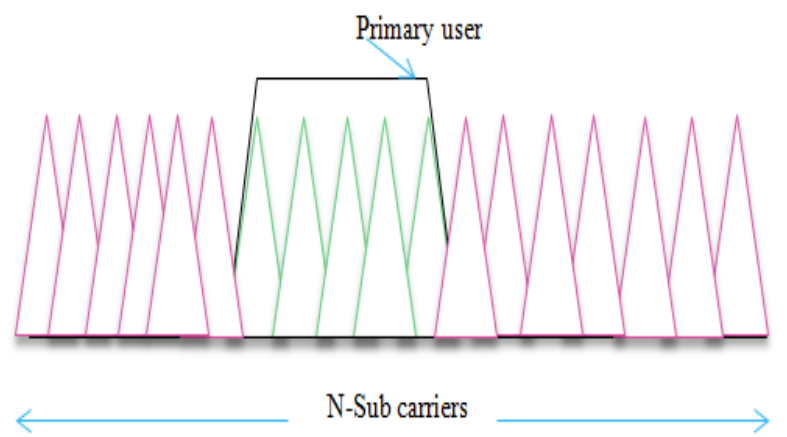

Fig. 3: Schematic of Primary and OFDM Signals.

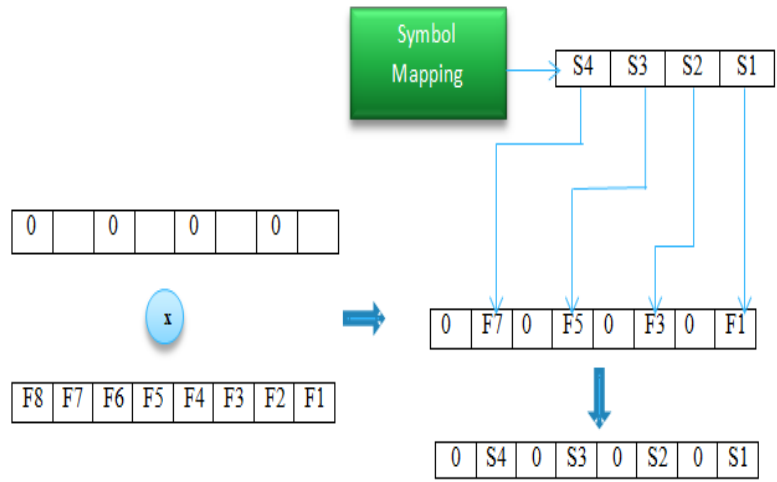

Fig. 4: Sub-Carrier Selection Using CR.

Method 2 of CR- OFDM is a spectral adaptation technique, in which $\mathrm{CR}$ scans the spectrum availability; wherever the primaries are unoccupied there the OFDM sub-carriers access the spectrum. The channel availability is varied according to time and for each time $\mathrm{CR}$ senses the idle channel $(\mathrm{CH})$. In Figure 5, PSD of primary Users (PUs) with OFDM sub-carriers is shown. In that 7 channels are available. Primary users occupy 2nd, 3rd, 4thand 7thchannels. CR senses the spectrum availability, after sensing, OFDM sub-carriers are allocated to the available spectrum1, 5 and 6 without causing interference to primary. Continuity of OFDM sub-carriers are not maintained, but still OFDM sub-carriers can be properly received, this is the main advantage of CR-OFDM. In this method no need to turn -off any sub-carriers of OFDM.

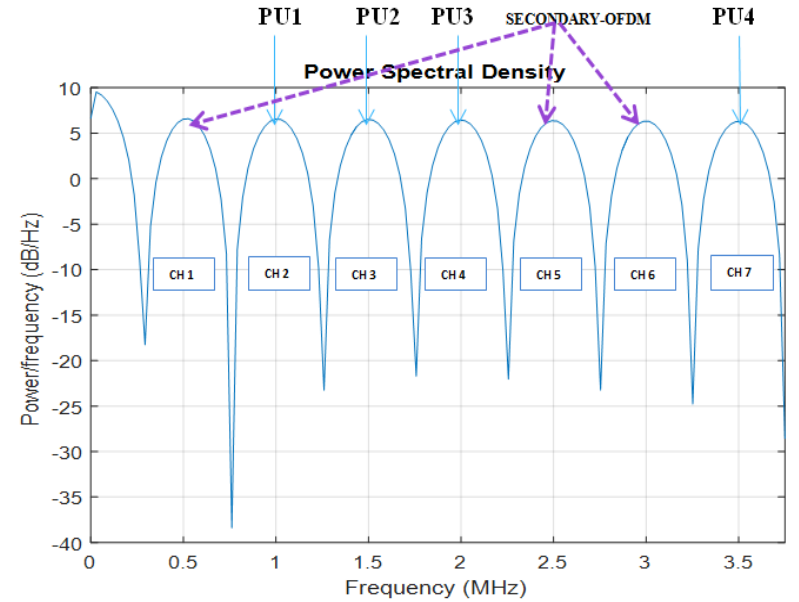

Fig. 5: PSD of Primary Users (Pus) with OFDM Sub-Carriers as Secondary. 


\section{CR-UWB}

In UWB, spectrum sensing is not required, because power usage is less than the noise margin, through which the UWB signal can be transmitted along with the presence of primary or secondary users. This typical type of parallel communication in the presence of primary is named as underlay. Block diagram of CR-UWB is illustrated in Figure 6, in which UWB pulse train is modulated using Binary phase shift keying (BPSK) and mixed with pseudo noise (PN) sequence. Then the spread sequence is transmitted through CR for channel allocation. In the receiver section, again the process is reversed to recover the original UWB pulses.

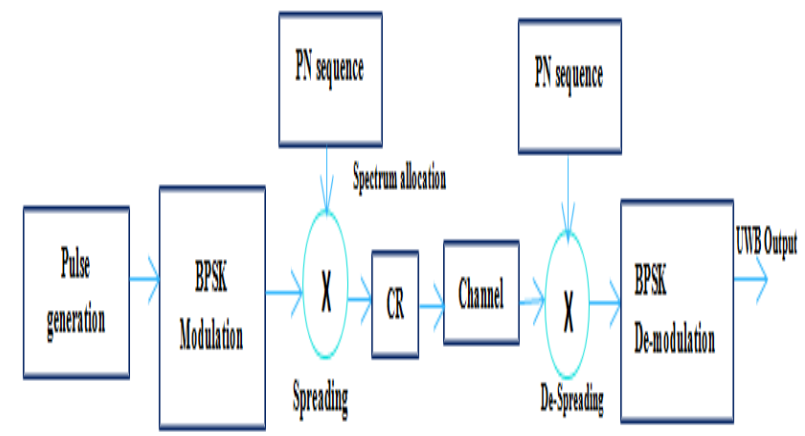

Fig. 6: Block Diagram of CR-UWB.

Bandwidth of UWB signal should be greater than or equal to 500 $\mathrm{MHz}$ with $-41 \mathrm{~dB} / \mathrm{MHz}$ transmitter power, and the applicable range of radio frequency in unlicensed band is between 3.1-10.6 GHz. This unique feature of CR-UWB rides the path towards green communication.

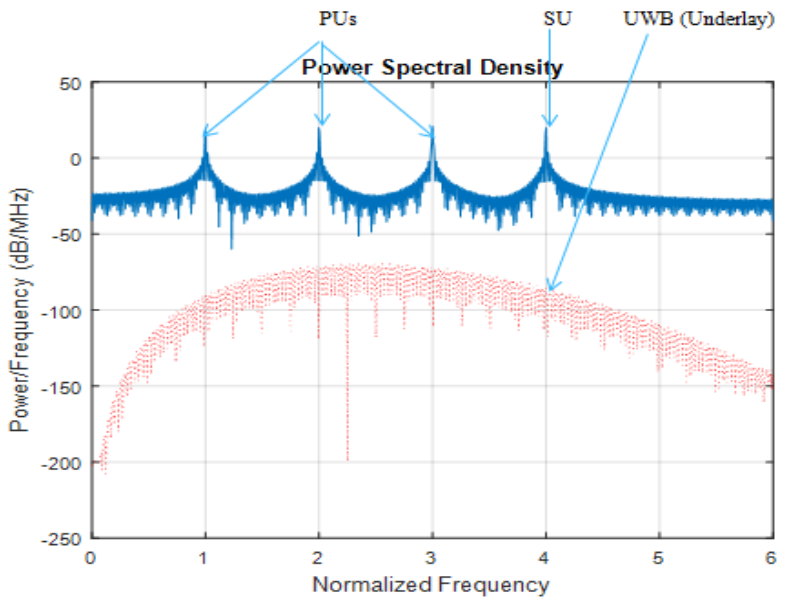

Fig. 7: PSD of Primary Users (Pus) with UWB.

In the above Figure 7, five channels are available. PUs occupies first, second and third channels and a single SU occupies the fourth channel UWB signal is transmitted along with PUs and SUs in the form of underlay.

\section{Conclusion}

In our proposed system $\mathrm{CR}$ cycle is enhanced to green cognitive cycle through which maximum efficiency can be achieved with minimum delay. By using CR-OFDM in a proper way, the resources are effectively utilized for spectrum allocation.CR-OFDM occupies the idle band of spectrum and masks (turned off) the intermediate sub-carriers in the position where primary users are available. UWB signal can transmit with minimum power and utilize the band without sensing the spectrum, so the sensing time gets reduced and parallel transmission is also possible in the presence of primary and secondary users. Hence the above said tech- niques are the best possible approach to achieve green communication.

\section{Acknowledgements}

The authors would like to acknowledge SASTRA Deemed to be University for the great support and assistance rendered to carry out this research work.

\section{References}

[1] Mitola J, M. G. "Cognitive radio: Making software radios more personal”, IEEE Personal Communications, 1999.

[2] S. Haykin, "Cognitive radio: Brain-empowered wireless communications", IEEE J. Sel. Areas Commun., vol. 23, no. 2, pp. 201-220, Feb.2005.

[3] A. Sharma and C. R.Murthy, "A group testing based spectrum hole search using a simple sub-Nyquist sampling scheme," in Proc. GLOBECOM, pp. 1526-1531, 2012.

[4] Y. C. Liang, K. C. Chen, G. Y. Li, and P. Mahonen, "Cognitive radio networking and communications: An overview", IEEE Trans. Veh. Technol., vol. 60, no. 7, pp. 3386-3407, Sep. 2011.

[5] T. Yucek and H. Arslan, "A survey of spectrum sensing algorithms for cognitive radio applications",IEEE Commun. Surv.Tuts., vol. 11 no. 1, pp. 116-130, First Quart., 2009.

[6] Y. Zeng, Y. C. Liang, A. T. Hoang, and R. Zhang, "A review on spectrum sensing for cognitive radio: Challenges and solutions", EURASIP J. Adv.Signal Process., vol. 2010, pp. 381 465-1-381 465-15, Jan. 2010.

[7] E. Axell, G. Leus, E. G. Larsson, and H. V. Poor, "Spectrum sensing for cognitive radio: State-of-the-art and recent advances", IEEE SignalProcess. Mag., vol. 29, no. 3, pp. 101-116, May 2012.

[8] Trigui, E.; Esseghir, M.; Boulahia, L.M., "Spectrum handoff algorithm for mobile cognitive radio users based on agents' negotiation", Wireless and Mobile Computing, Networking and Communications (WiMob), 2013 IEEE 9th International Conference, vol., no., pp.750, 756, 7-9 Oct. 2013.

[9] Lu Li; YanmingShen; Keqiu Li; Kai Lin, "TPSH: “A Novel Spectrum Handoff Approach Based on Time Estimation in Dynamic spectrum networks"," Computational Science and Engineering (CSE), 2011 IEEE 14th International Conference, vol., no., pp.345,350, 24-26 Aug. 2011.

[10] Christian, I; Moh, S.; Ilyong Chung; Jinyi Lee, "Spectrum mobility in cognitive radio networks",Communications Magazine, IEEE, vol.50, no.6, pp.114,121, June 2012 doi:10.1109/MCOM.2012.6211495

[11] M Matthe, LL Mendes, I Gaspar, N Michailow, D Zhang, G Fettweis, "Multi-user time-reversal STC-GFDMA for future wireless networks",EURASIP J. Wirel. Commun. Netw, 2015, 132 (2015).

[12] M Taherzadeh, H Nikopour, ABayesteh, H Baligh, in Proc. IEEE VTC-Fall. CMA codebook design, (2014).

[13] Mohandass Sundararajan and Umamaheswari Govindaswamy, "Multicarrier Spread Spectrum Modulation Schemes andEfficient FFT Algorithms for Cognitive Radio Systems" Electronics 2014, 3 , 419-443; doi: 10.3390/electronics3030419 (2014). 